\title{
Pulsed Electrodeposition \\ of Carbon Dioxide Reduction Electrocatalysts for Space Applications
}

Brian Skinn $^{a}$, Sujat Sen ${ }^{b}$, McLain Leonardc, Dan Wanga,

Fikile Brushett ${ }^{c}$, EJ Taylora

a Faraday Technology, Inc., Englewood, OH, USA 45315

b Dept. of Chemistry \& Biochemistry, University of Wisconsin, La Crosse, WI, USA 54601

c Dept. of Chemical Engineering, Massachusetts Institute of Technology, Cambridge, MA, USA 02139

16 Oct 2019

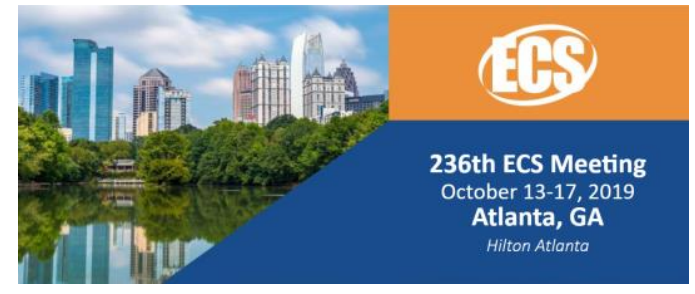

BrianSkinn@FaradayTechnology.com

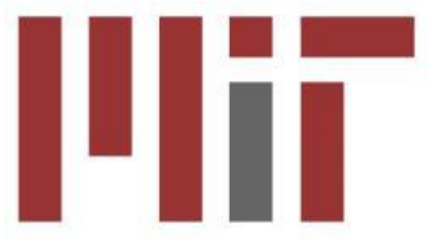




\section{Terrestrial Motivation}

- $\mathrm{CO}_{2}$ Conversion

- Environmental concerns

- Abundant carbon feedstock for valueadded products

- CO, formate, hydrocarbons

- Point sources (e.g., power generation)

- Co-location of capture \& conversion

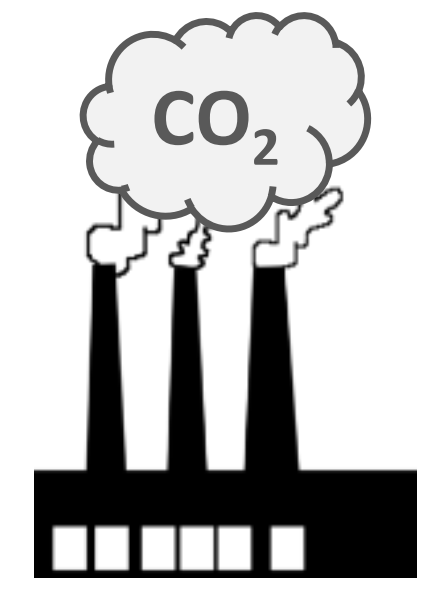

Value-Added Chemicals

- Direct conversion of gas-phase $\mathrm{CO}_{2}$

- Gas-diffusion electrode (GDE) electrolyzer 


\section{NASA Relevance}

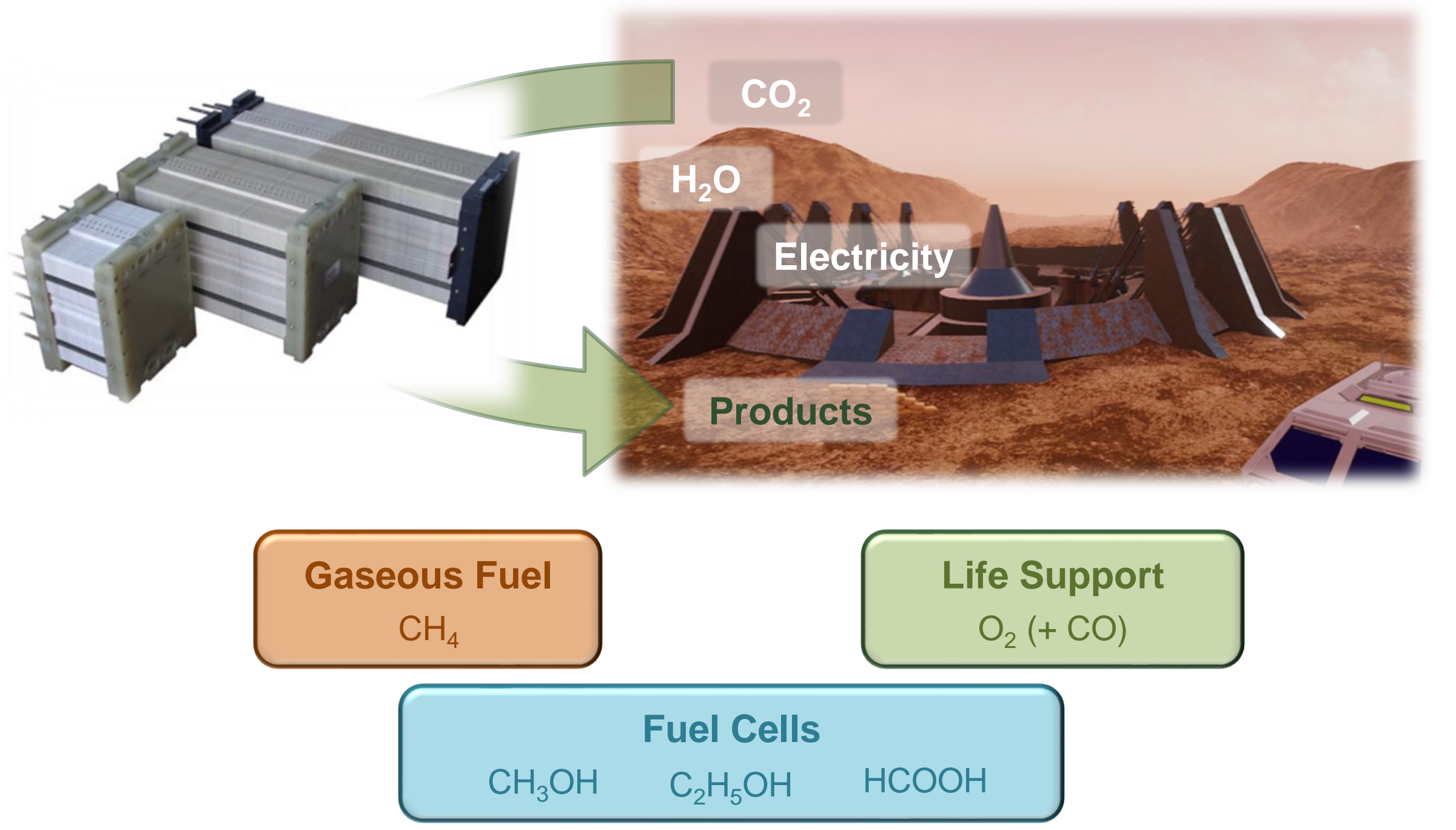

FARADAY תחת ת. TECHNOLOGY, INC. 


\section{Pulse/Pulse-Reverse Electrodeposition}

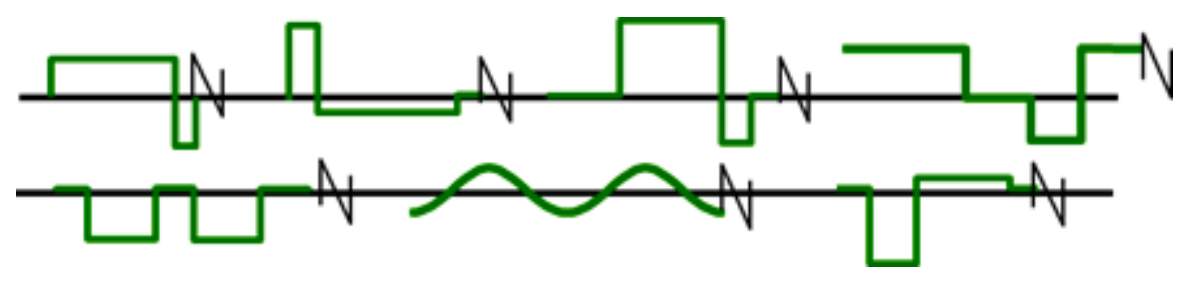




\section{Prior Work: Lead-Free Tin}

\section{Rotating Disk Plating Tool}

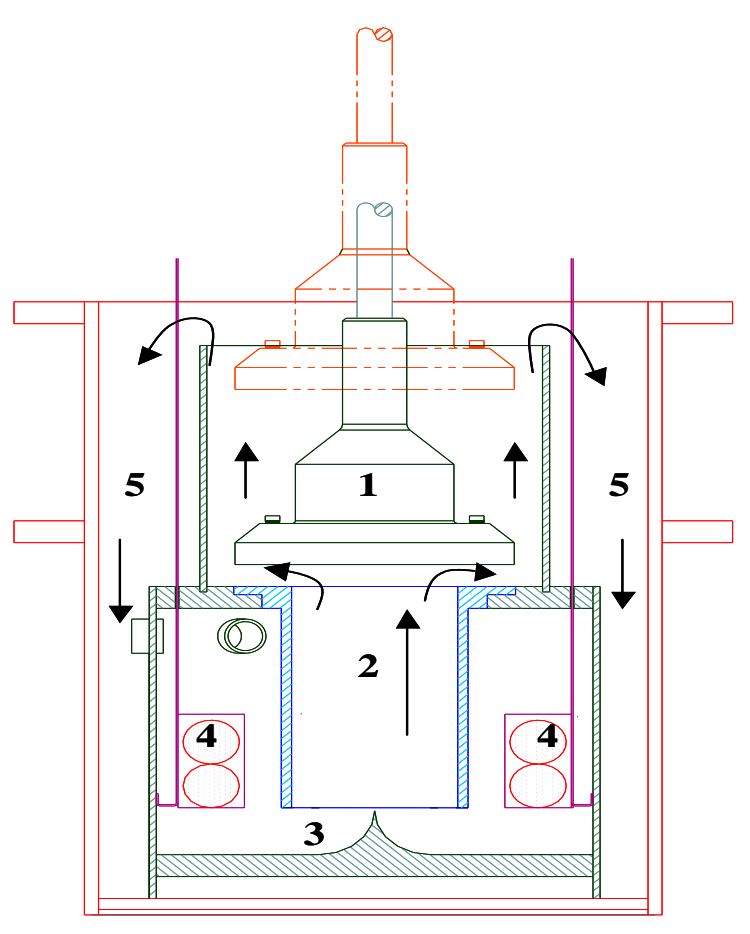

FARADAY ЛПЛЛ TECHNOLOGY, INC.
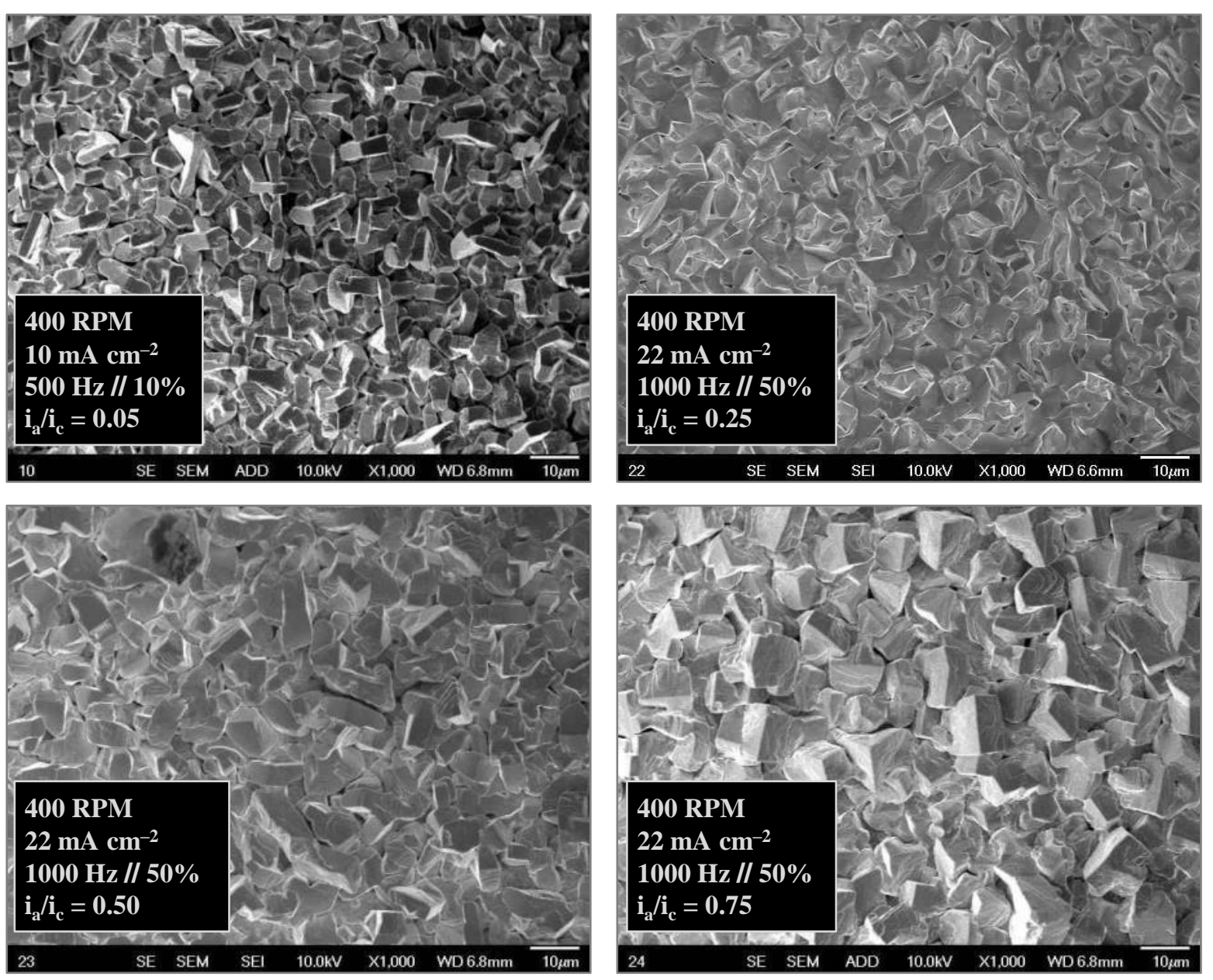

Sn Deposits on Cu Substrates - 1000x Magnification U.S. Patent 8,603,315 Granted 


\section{Prior Work: Copper Electrodeposition for PCBs}

\section{Standard DC Recipe Pulsed Waveform Recipe}

$\mathrm{CuSO}_{4}$

"Accelerator"

"Suppressor"

"Leveler"

Other Additives
Z-Interconnects Cu-Plated by Pulse-Reverse FARADAYIC ${ }^{\circledR}$ ElectroDeposition
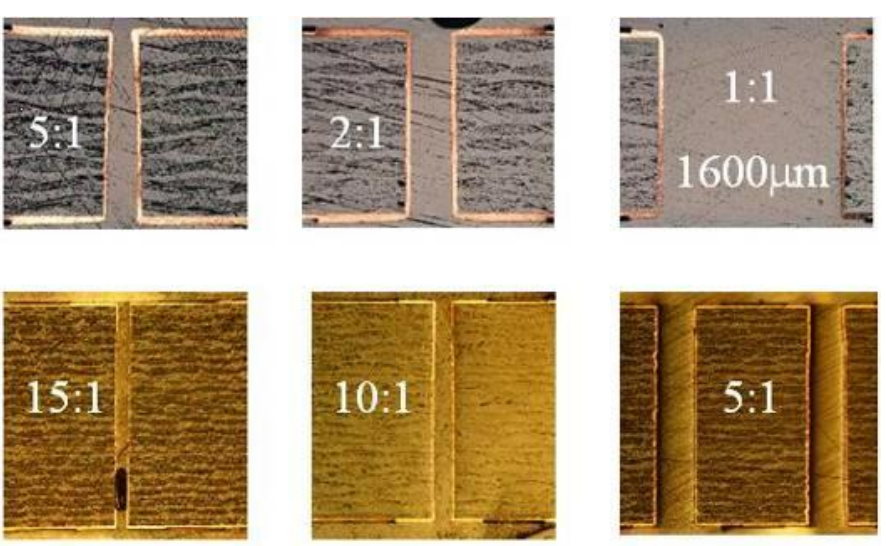

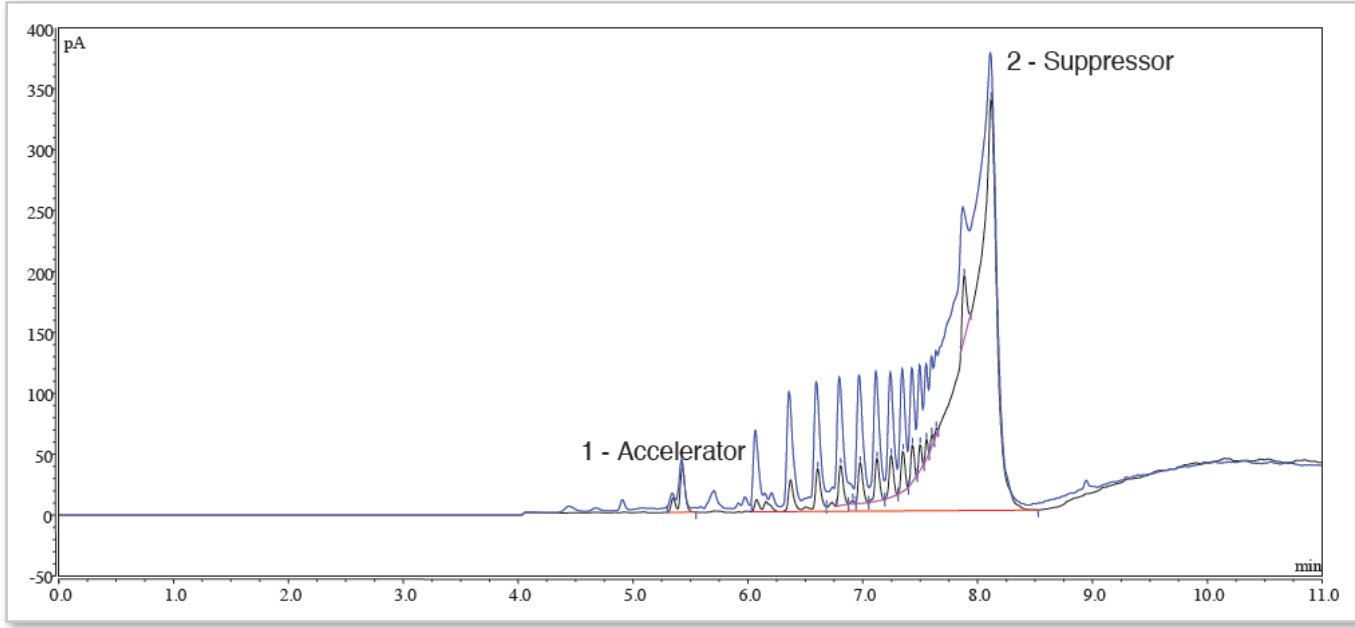

Plante, et al. "Quantization and Characterization of Copper Plating Bath Additives by Liquid Chromatography with Charged Aerosol Detection." Thermo Scientific. http://www. dionex.com/enus/webdocs/113469-Pittcon12 1820-6 MPlante PN70008 PlatingBaths.pdf

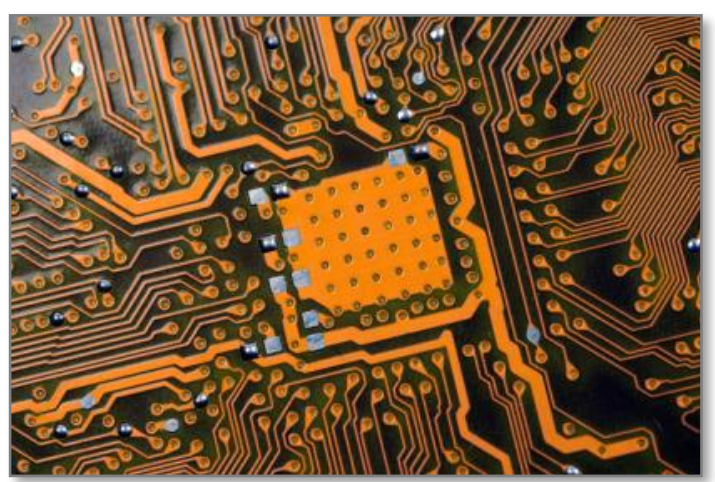

FARADAY ת几Л TECHNOLOGY, INC. 


\section{Prior Work: NiMo Functionally-Graded Coating}

Functionally graded NiMo coatings for improved corrosion resistance in moltenfluoride-salt nuclear reactors (MSRs)

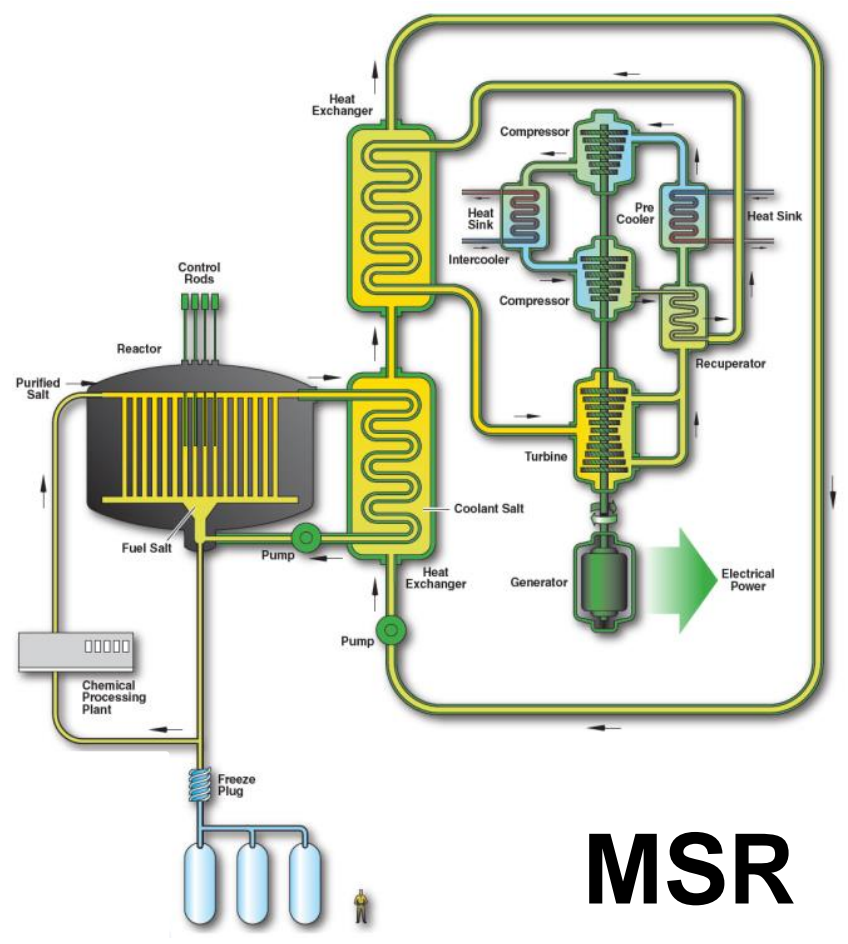

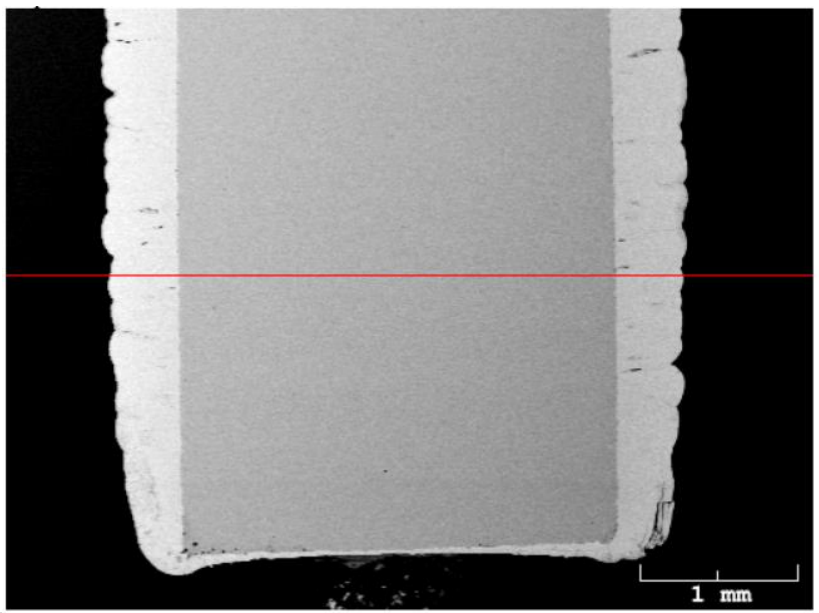

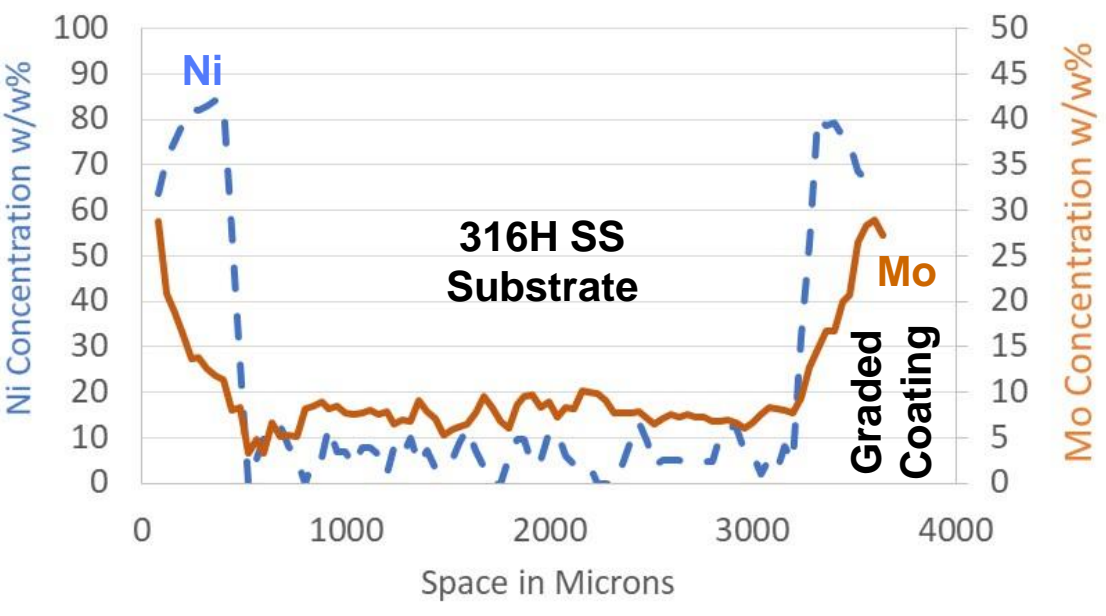




\section{FARADAYIC ${ }^{\circledR}$ ElectroCatalyzation}

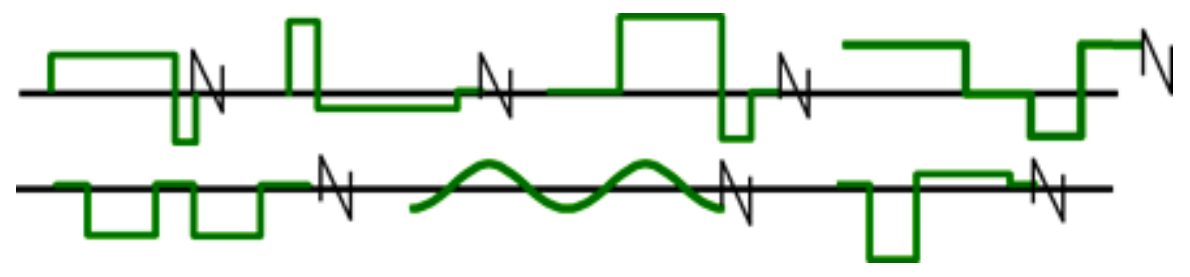

Gas Diffusion Electrode (GDE) Electrocatalysts 


\section{FARADAYIC ${ }^{\circledR}$ GDE ElectroCatalyzation}

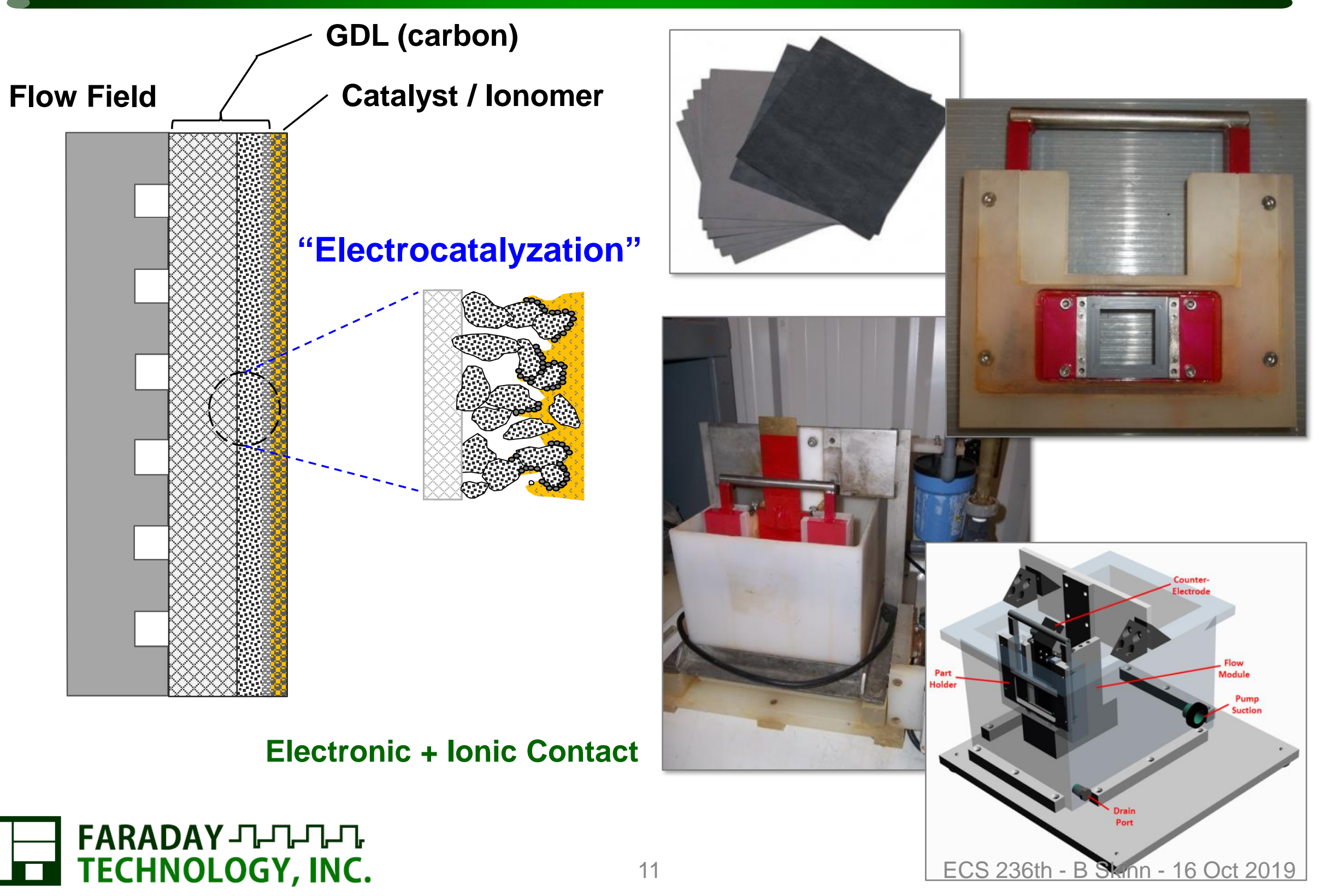




\section{$\mathrm{CO}_{2}$ GDE Catalyst Product Selectivity}

Product distribution over single-metal electrocatalysts as determined by Hori et al. Electrochimica Acta 39(11-12): 1833 (1994).

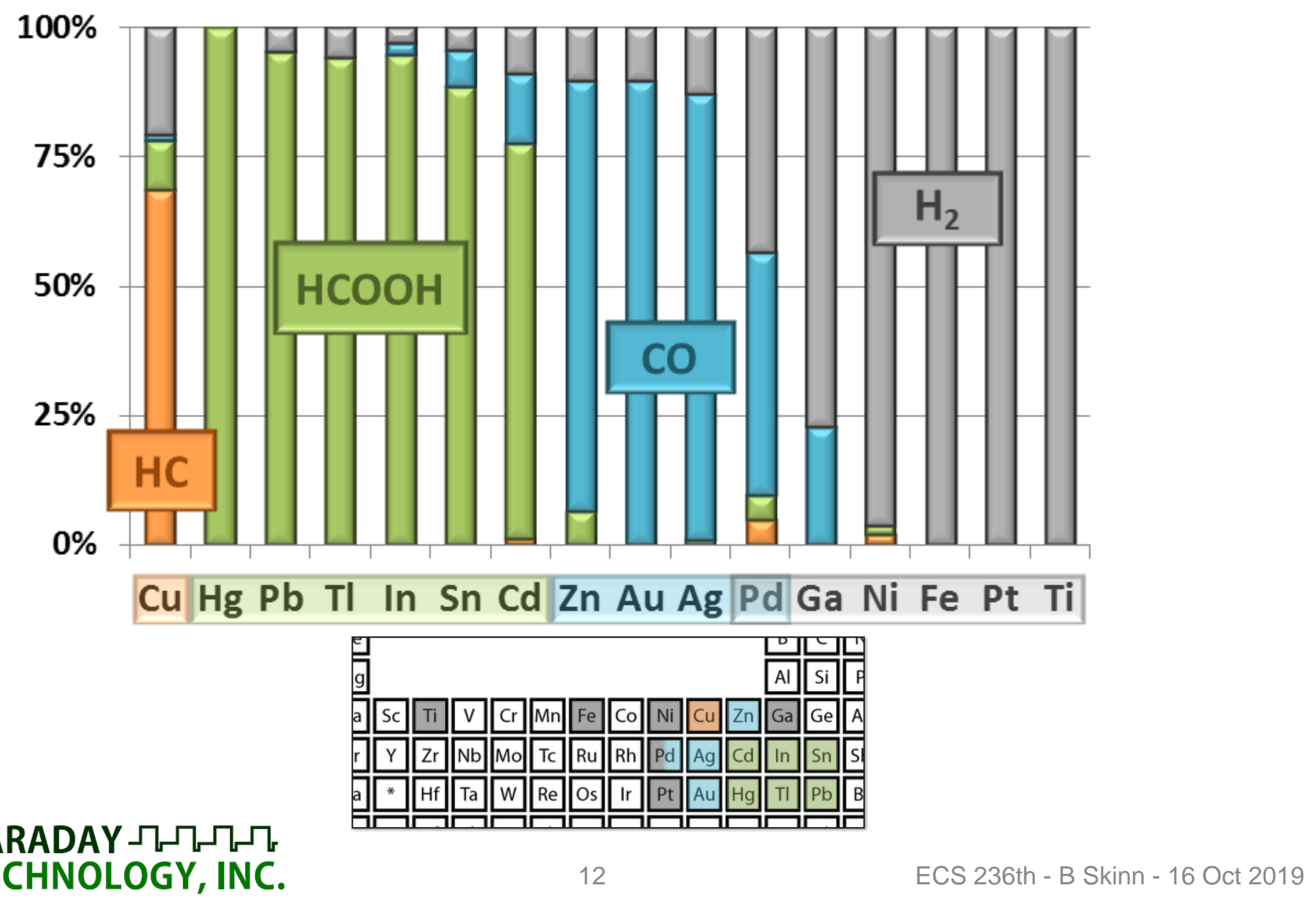




\section{$\mathrm{CO}_{2}$ GDE Catalyst Product Selectivity}

Shifts in product distribution over copper-alloy electrocatalysts vs pure Cu as reviewed by Nitopi et al. Chemical Reviews 119: 7672 (2019).
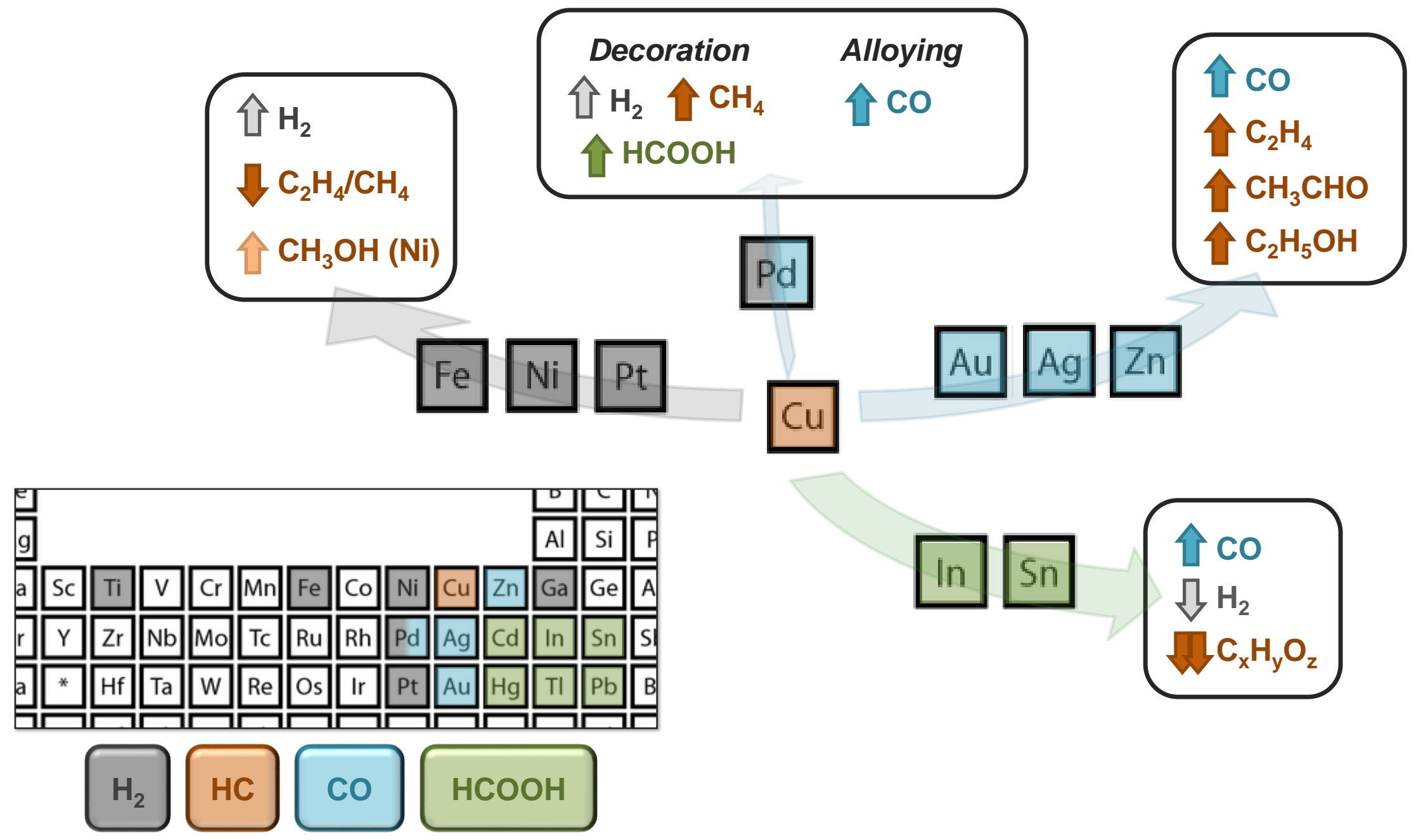

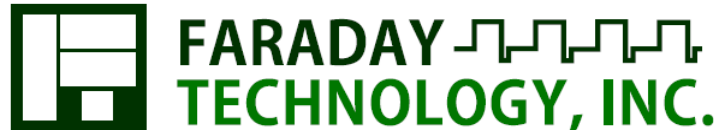




\section{$\mathrm{CO}_{2}$ GDE Catalyst Product Selectivity}

Product distribution over single-metal electrocatalysts as determined by Hori et al. Electrochimica Acta 39(11-12): 1833 (1994).

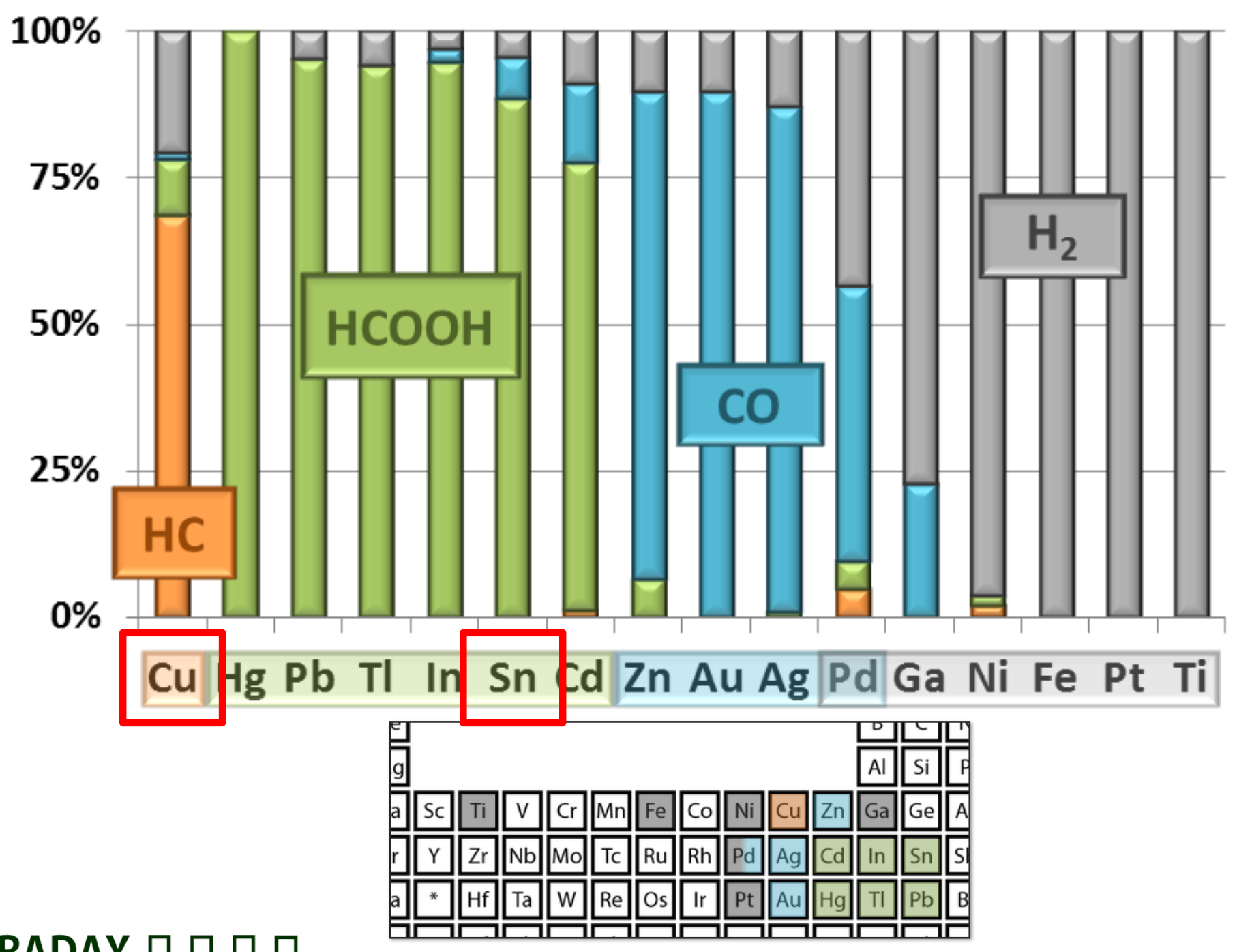

FARADAY תЛЛ TECHNOLOGY, INC. 


\section{GDE Catalysts - Sn}
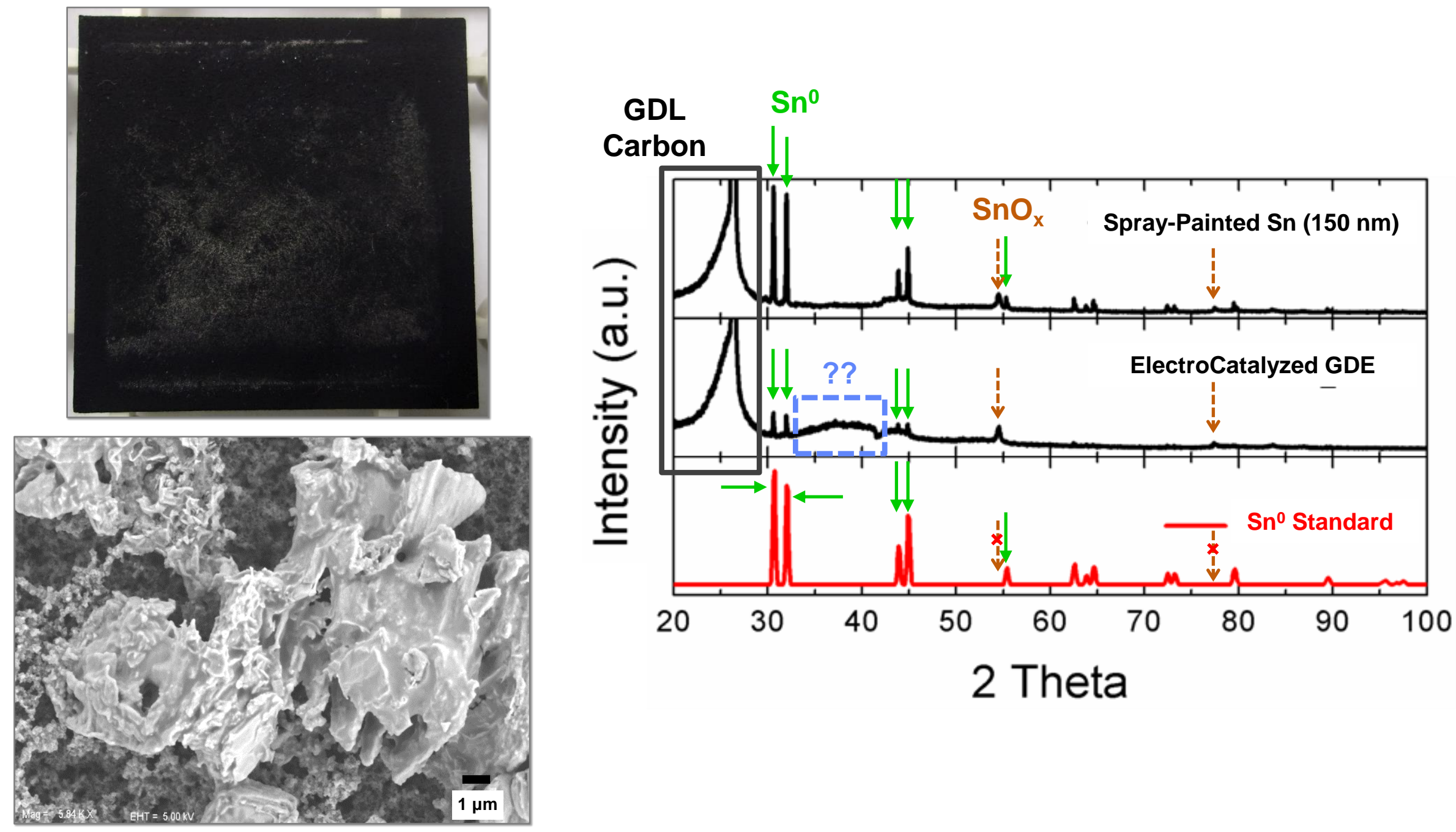

FARADAY ת תחת TECHNOLOGY, INC. 


\section{GDE Catalysts - Cu}
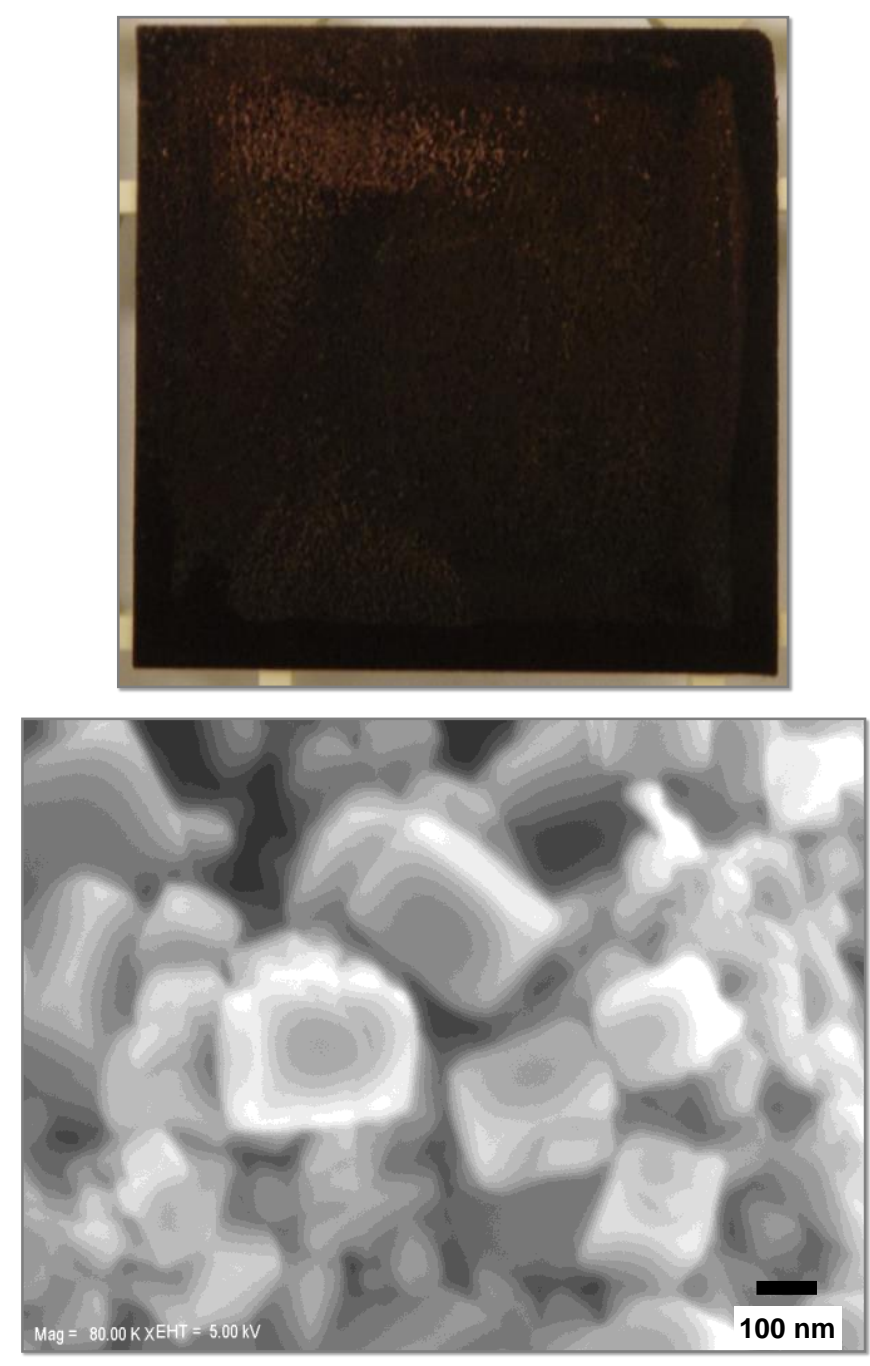

FARADAY תЛП TECHNOLOGY, INC.

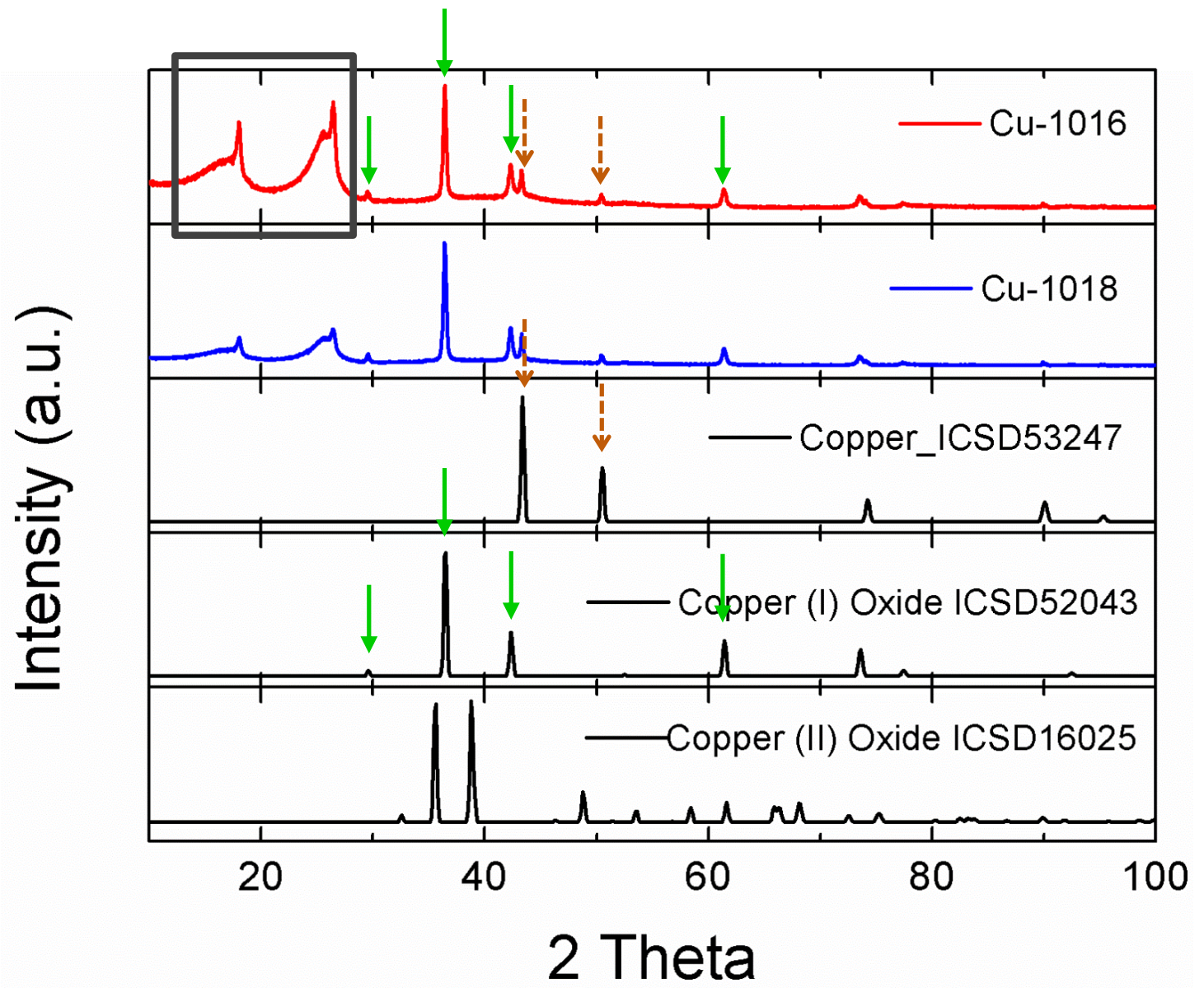




\section{Electrocatalyst Testing}

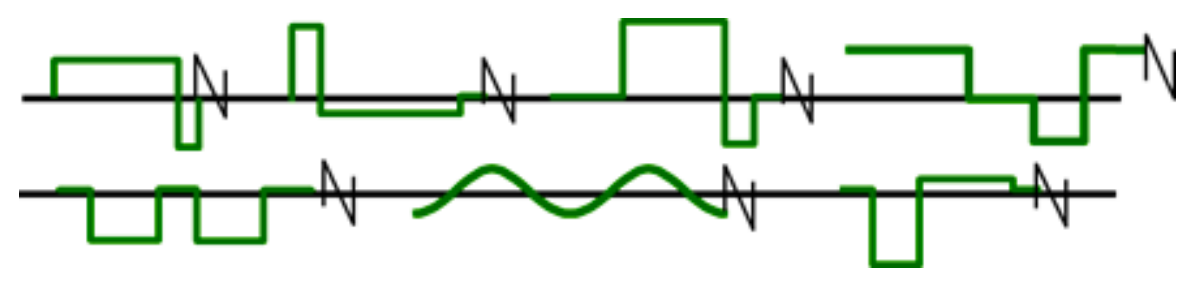




\section{W-Cell Apparatus}
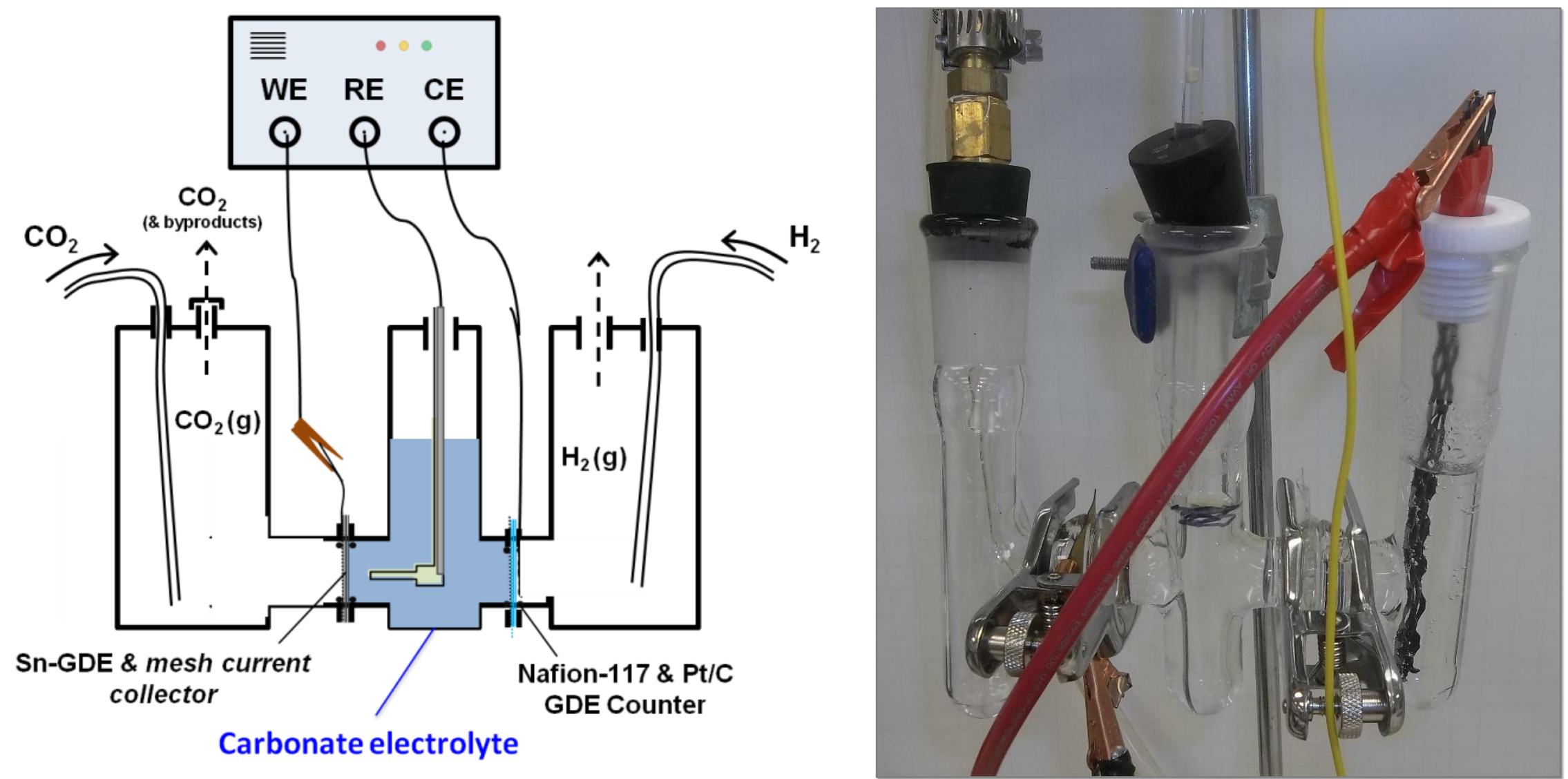

FARADAY תЛת TECHNOLOGY, INC. 


\section{Flow Cell Apparatus}

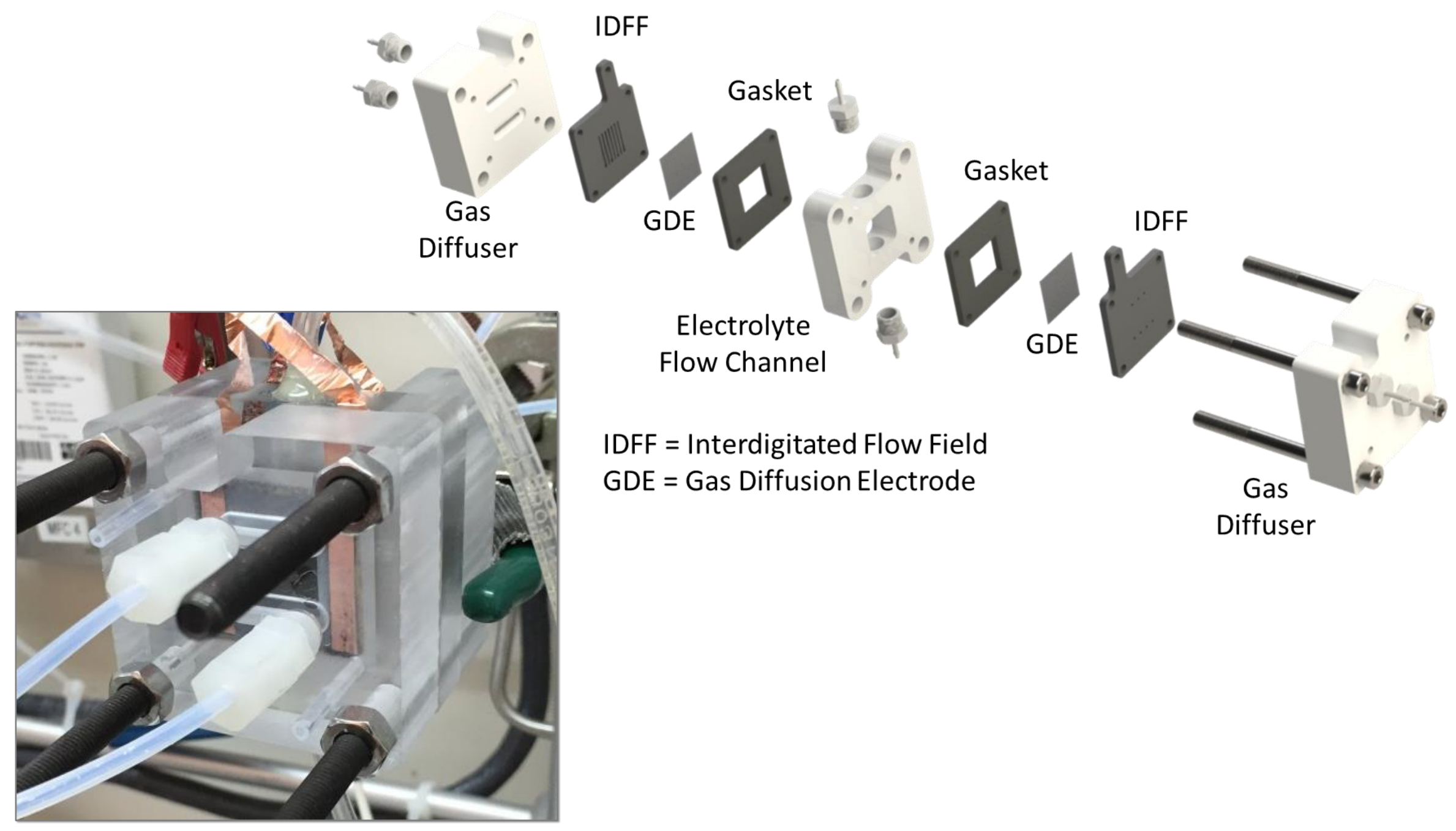

FARADAY ת ת TECHNOLOGY, INC. 


\section{Sn GDE - W-Cell Electrocatalysis}
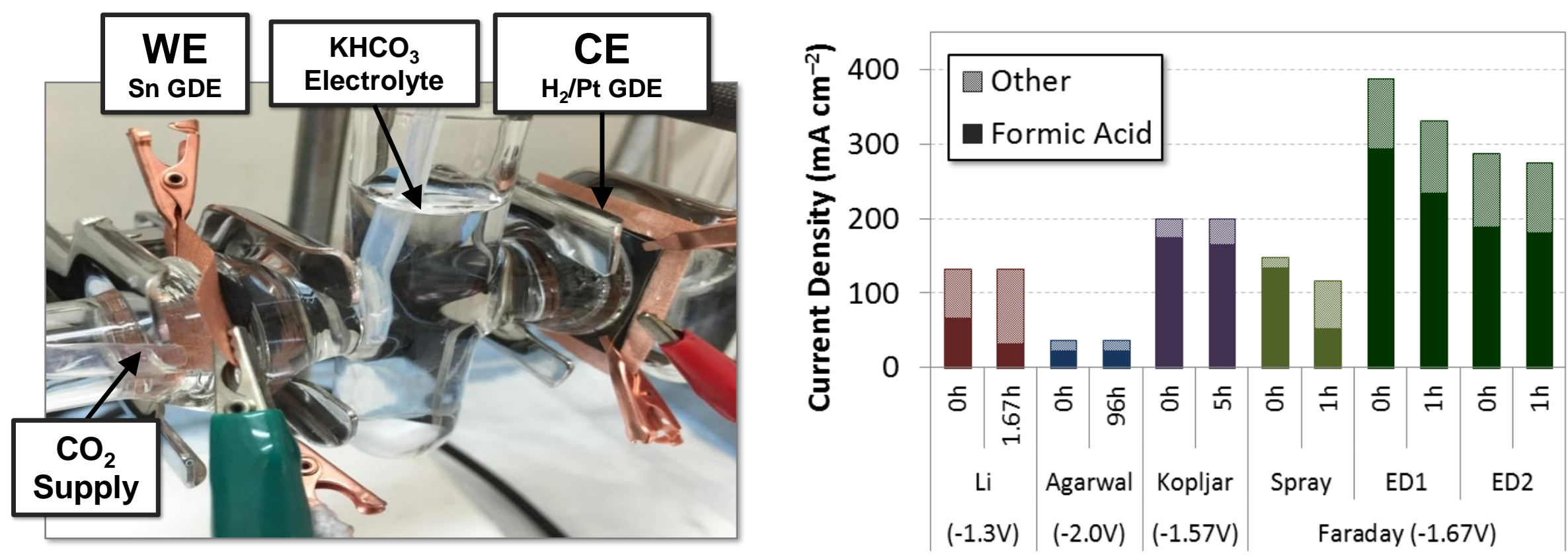

(Half-cell potentials vs SHE)

WE: $\mathrm{CO}_{2}+2 \mathrm{H}^{+}+2 \mathrm{e}^{-} \rightarrow \mathrm{HCOOH}$

CE: $\quad \mathrm{H}_{2} \rightarrow 2 \mathrm{H}^{+}+2 \mathrm{e}^{-}$

Li and Oloman. J Appl Electrochem 35: 955, 2005.

Agarwal et al. ChemSusChem 4: 1301, 2011.

Kopljgar et al. J App/ Electrochem 44: 1107, 2014.

Cell: $\quad \mathrm{CO}_{2}+\mathrm{H}_{2} \rightarrow \mathrm{HCOOH}$ 


\section{Cu GDE - Flow Cell Electrocatalysis}
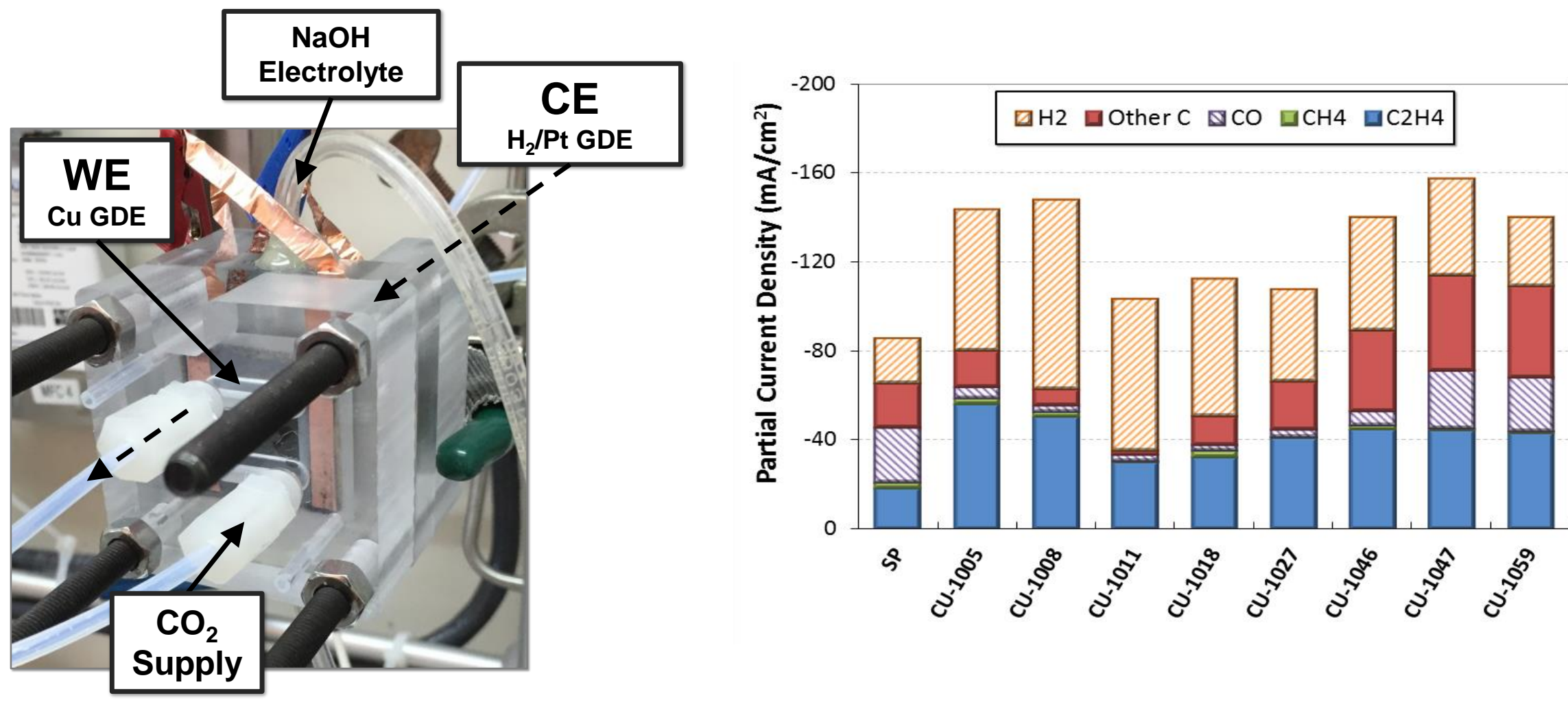

WE: $2 \mathrm{CO}_{2}+8 \mathrm{H}_{2} \mathrm{O}+12 \mathrm{e}^{-} \rightarrow \mathrm{C}_{2} \mathrm{H}_{4}+12 \mathrm{OH}^{-}$

CE: $\quad 12 \mathrm{OH}^{-} \rightarrow 6 \mathrm{H}_{2} \mathrm{O}+3 \mathrm{O}_{2}+12 \mathrm{e}^{-}$

Cell: $\quad 2 \mathrm{CO}_{2}+2 \mathrm{H}_{2} \mathrm{O} \rightarrow \mathrm{C}_{2} \mathrm{H}_{4}+3 \mathrm{O}_{2}$

FARADAY תЛת TECHNOLOGY, INC.

Sen, S. et al. "Pulse Plating of Copper onto Gas Diffusion Layers for the Electroreduction of Carbon Dioxide." MRS Adv 3(23): 1277 (2018). 


\section{Summary}

- Wide variety of active GDE catalyst materials can be deposited via pulsed FARADAYIC ${ }^{\circledR}$ ElectroCatalyzation

- Readily scalable process

- High-activity $\mathrm{CO}_{2}$ conversion catalysts

- Demonstrated in industrially-relevant, scalable form factor

- Performance competitive with or superior to literature

- Total activities $\left(\mathrm{mA} / \mathrm{cm}^{2}\right)$

- Formate/ethylene selectivity

- Durability appears promising; further testing is pending

- Reactor/GDL development in progress

- Goal: $100+$ to $1000+$ hours of steady operation

- Goal: Optimization of Sn ElectroCatalyzation parameters 


\section{Acknowledgment}

- This presentation is based upon work supported by the United States Department of Energy (contracts \#DE-SC0015173 and \#DE-SC0017199).

- Contact Information:

Brian Skinn

Ph: +1937836 7749

Email: BrianSkinn@FaradayTechnology.com

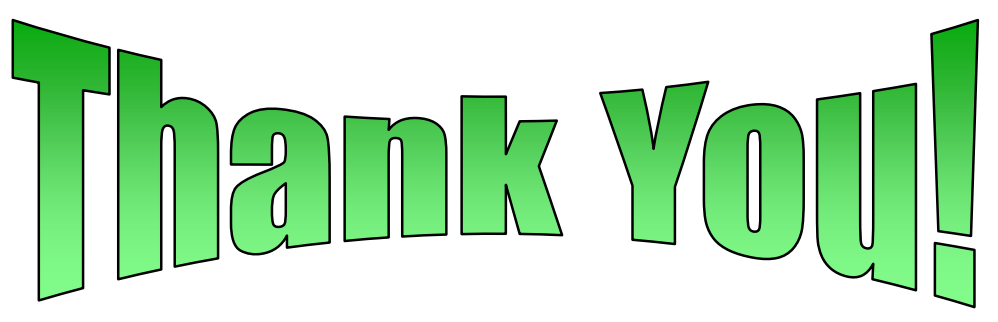

